\title{
Glomerulopathy Associated with Parasitic Infections
}

\author{
M-L.F. van Velthuysen
}

Numeroti: infectious diseases, anong them several parasitic infections, have beeil shown to be associated woith glomerular disease, although the exact pathogenetic mechanisms lave not yet been elucidated. In this article, Marie-Lowisi vin Velthuysen reviews the work published on glomertiopathy associated with the most important parasitic in ficicns, ie. malaria, schistosomiasis, leishmaniasis and irupnosomiasis.

The pathogenesis of glomerulonephritis is often thought to be immunologically mediated, because in many instancer, glomerular changes are seen with immunoglohulin depositions. It is interesting that the incidence of the nephrotic syndrome and chronic renal failure: is much higher in tropical areas than in temperate climates ${ }^{1}$. This suggests that parasitic infections such as malaria might be involved in the pathogenesis of glonerular disease in tropical areas, but probably host-related factors, determining the susceptibility for the development of glomerular lesions, are involved as well 2,3 .

Recent advances in the knowledge of the immunopathogenesis of glomerular disease show that similar mechanisms may be involved in the pathogenesis of primary (idiopathic) and secondary glomsrulopathy (infection-associated glomerulopathy).

\section{Malaria}

Malaria was the first parasitic infection that was clearly shown to be associated with the nephrotic syndrome in tropical areas ${ }^{1,5}$. This is especially true for quartan malaria (Plasmodium malarine), although renal involvement in falciparum malaria (Plasmotium falciparum) has also been described". Quartan malaria is associated with chronic glomerular disease which is usually not reversible by treating the infection? Renal involvement in falciparum malaria is usually transient, and disappears when the infection is brought under contrcl. In some instances, however, persistent glomerular lesions were described. The glomerular lesions associated with $P$. falciparum infection are thought to be due to general mechanisms such as hypovolemia, ischemia and intravascular coesgulation. In both infections ( $P$. malariae and $P$. falciparum) histological glomerular lesions are variable, with irregular thickening of the glomerular capillary wall, and proliferative glomerular changes. In quartan malaria, segmental sclerosis of the glomerular tuft can be seen as well, sometimes leading to global glomerulosclerosis. Immunofluorescence shows fine and coarse granular mesangial staining for IgM, IgG and C3 with some extensions along the glomerular capillary loop ${ }^{8}$. On the ultrastructurati level, electron-dense deposits are seen in the mesangial and subendothelial areas.

Marie-Louise van Velthuysen is at the Department of Pathology, Uniralsity of Rotterdam, PO Box 1738, 3000 DR Rotterdam. The Netherlands. Tel: +31 10408 792.4, Fax: +31 10436 6660, e-mail: vanVelthuysen@path.AZR.NL
The glomerular immunoglobulins were initially thought to originate from circulating immune complexes as these could be easily detected ${ }^{9,10}$. To explore the pathogenetic mechanisms involved in the development of this glomerular disease, animal models were developed. Some research focused on Plasmodium infections in monkeys and chicken, but most work was carried out in rodents, especially in mice using Plasmodium berghei infection. Mice infected with $P$. berghei developed a glomerular disease charanterized by mild expansion of the mesangial matrix, and mesangial staining for immunoglobulins"1. Mild transient proteinuria was detected ${ }^{12}$. Malarial antigen was seen in glomeruli three days after infection, while immunoglobulins were detected only after seven days ${ }^{1}$, suggesting in situ immune-complex formation. Similar results were obtained in a rat model ${ }^{13}$ As the glomerular disease was only mild and transient, these experimental models were not a suitable representation of the human counterpart of glomerular involvement in quartan malaria. Similarities with the glomerulopathy associated with falciparum malaria, however, were suggested ${ }^{12,13}$. A satisfactory explanation for the persistent glomerular lesions associated with quartan malaria was not found. In later studies, proliferative glomerular changes with overt proteinuria were obta'ned in mice ${ }^{14,15}$. It is not clear whether this difference in occurrence of glomerular disease is due to more-sensitive detection methods or to the use of different parasite or mouse strains. The variation between mouse strains could be relevant to study the differences in host susceptibility (see above). Moreover, these studies suggested a role for DNA-binding antibodies and cell-mediated immune mechanisms in the development of nephritis during murine malaria.

Thus, glomerular lesions can be associated with malaria infection. Murine models have shown that humoral as well as cellular immune mechanisms are apparently involved in the pathogenesis of these lesions. Apart from immunological mechaisisms, local release of inflammatory mediators and disiurbance of the microvasculature by intravascular coagulation could participate by damaging of glomerular endothelium. Future studies should elucidate the exact mechanisms involved.

\section{Schistosomiasis}

Although schistosomiasis is one of the old the most widespread parasitic infections, its ass ticition with glomerular disease was established onty in the 1970s. Hepatosplenic schistosomiasis (induced by Schistosoma mansoni), in particular, ivas shown to be associated with glomerular changes and renal failure ${ }^{16-18}$. In cases of glomerular disease associated with Schistosoma haematobium infection, concomitant chronic salmonellosis seemed to be involved ${ }^{18}$. Overall incidence of glomerular disease with schistosomiasis was shown to be about $5 \cdots 6 \%$, whereas in patients with hepatosplenic disease due to $S$. mansoni 
infection glomerulonephritis was seen in $15 \%$ of patients 1 .

The pathological changes in glomeruli of patients infected with S. mansomi are variable as in Plasurditum infection. The most common glomerular lesions accompanying schistosomiasis are mesangioproliferative and membrano-proliferative changes, but membranous glomerulopathy, focal sclerotic glomernlar lesions and rapidly progressive glomerulonephritis are occasionally geen as well ${ }^{16,17,19}$. With immunofluorescence, immunoglobulins (IgM, IgG, Ig, A and IgE) and complement components are observed in the glomerular mesangium with small extensions along the capillary loops ${ }^{20,21}$. On the ultrastructural level, electrondense deposits (suggesting immune complexes) are seen in the mesangium and along the endothelial side of the glomerular basement membrane ${ }^{17}$. Sometimes renal histological changes were found to precede clinical manifestations ${ }^{17}$.

The glomerular disease associated with schistosomiasis was thought to be a typical example of immunecomplex glomerulonephritis. The presence of schistosomal worm antigens in the glomerular complexes $22-2.4$, and the presence of circulating immune complexes containing schistusomal antigen, supported this hypothesis. Later studies, however, did not show amelioration of the glomerular disease with treatment of the infection $2,2,2$ and the severity of the glomerular lesions did not seem to depend on the interisity of parasite infestation ${ }^{27}$. Moreover, Hillyer and Lewert ${ }^{2 *}$ found precipitating antibodies to DNA in sera from hamsters infected with $S$. joponicimm. This suggested a role for anti-DNA antibodies, in a context of polyclonal B-cell activation, as in systemic lupus erythematosus. On the other hand, Fujiwara and colleagues ${ }^{21}$ showed that polyclonal B-cell activation alone was not enough to induce glomerular disease in infected mice. They sugge: ted different, hostdependent factors, which are not yet defined.

Because glomerular changes are seen particularly in hepatosplenic schistosomiasis, portal-systemic shunting was thought to be associated with the development of glomerular lesions ${ }^{8}$. Experiments in mice, where ligation of the portal vein was performed, showed enhancement of immune-complex deposition in the kidney ${ }^{23}$. Glomerular lesions, however, were more marked and more frequent in hepatosplenic schistosomiasis than in active hepatic cirrhosis ${ }^{21}$. Therefore, factors other than shunting of portal blood into the systemic circulation alone had to be involved. The main difference between hepatosplenic schistosomiasis and cirrhosis is the presence of circulating schistosomal antigen. In addition, the severity of the glomerular lesions and proteinuria were shown to be correlated with the impairment of hepatic macrophage function ${ }^{30}$. This macrophage function might involve clearance of circulating immune complexes as well as clearance of other nephritogenic factors.

Thus, hepathosplenic schistosomiasis is clearly associated with glomerular disease, but a causal relation between glomerular lesions and parasites has been difficult to establish. Beside immune complexes, hostrelated factors, such? as hepatic macrophage function, seem to be involved.

\section{Leislımaniasis}

Glomerular lesions have been observed with visceral leishmaniasis (kala-azar) caused by Leishmmonin donownini. Cutaneous or mucocutaneous leishmaniasis caused by other Luishmanin species (tropicana, mexicamm, etc.) have not been associated with renal disense.

The glomerular lesions seen with kala-azar consist of mesangial expansion, sometimes with focal segmental proliferasunts". Using immunofluorescence, $\operatorname{IgG}, \operatorname{IgM}, \lg A$ and $C 3$ are seen in the mesangium, with some extensions along the capiliary loop ${ }^{31,32}$. On the ultrastructural level, irregular thickening of the glomerular basement membrane is seen with subendothelial and subepithelial electron dense deposits ${ }^{31}$. A prospective study has shown that $60 \%$ of patients with kala-azar have urinary abnormalities ${ }^{33}$. Renal biopsies demonstrated interstitial changes in all patients, while five out of seven patients had mild glomerular lesions, compatible with the lesions described earlier. In this study, renal involvement seemed to revert with the cure of the leishmanial infection. Experimental infu.ions with L. donorani in hamsters have resulted in a glomerular disease comparable with its human counterpart, although in hamsters renal amyloidosis was observed ${ }^{34,35}$. Since $L$. donovani antigens were detected in the glomerular lesions, these antigens were implicated in the pathogenesis of this glomerular disease ${ }^{32,34}$.

\section{Trypanosomiasis}

Although glomerular disease associated with Trypanosoma brucei infection has been described in 


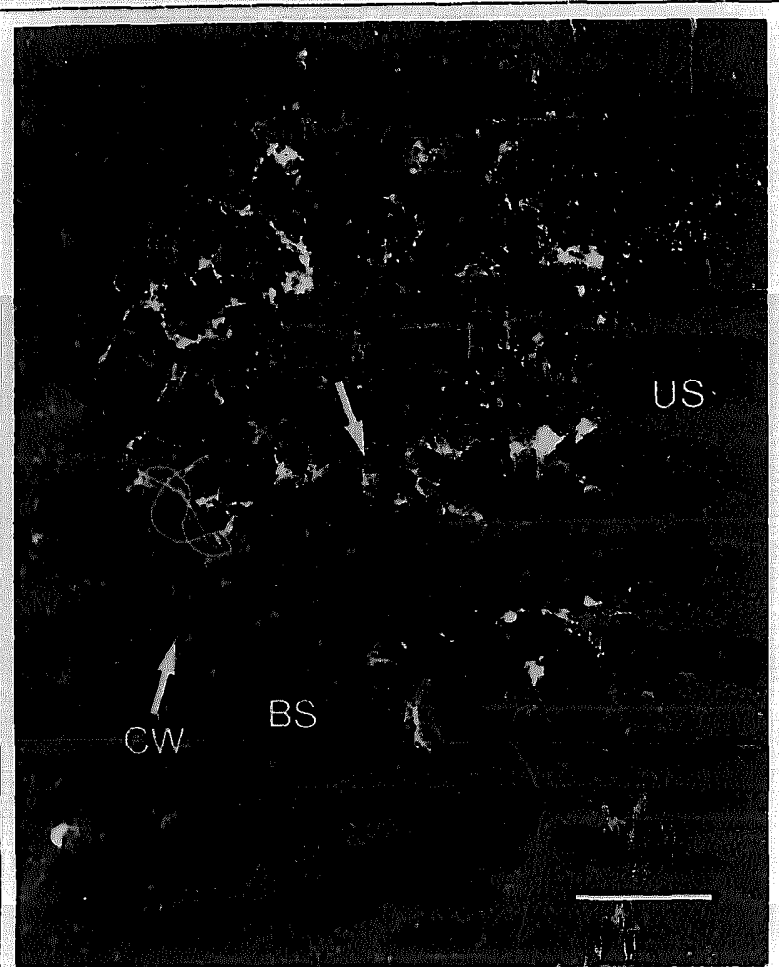

Fig. 2. Direct fluorescence staining for $\lg G$ in a mouse glomerulus six weeks after inoculation of Trypanosoma brucei brucei. BS, blood space; US, urinary space; CW. capillary wall; arrow points at the mesangium. Scale bar $=20 \mu \mathrm{m}$.

several species ${ }^{36-13}$, human glomerular disease associated with African trypanosomiasis is limited to an occasional reportt as is the association of Trypanosomm crusi infection (Chagas diseaso) with glomerulonephritists.

The glomerular disease associated with Atrican trypanosomiasis in animals, however, is of great incerest. The high prevalenee of African trypanesomiasis in cattle has prompted many to investigate faciors involved in resistance to the infection. The knowledge from these studies and the relative ease in inducing experimental disease allows study of the mechanisms involved in the pathogenesis of the associated giomerulopathy, which might be a model for infection related glomerulopathy in general.

Glomerular disease associated with $T$. brucei infection has been described in rhesus monkeys.30, rabbits $^{37,34}$, rats ${ }^{31-11}$ and nice ${ }^{2,43}$. The glomerular changes observed vary from mesangioproliferative changes to expansion of the mesangial matrix alone (Fig. 1). With inmumofluorescence IgM, IgG and complement components are seen in the mesangium, witi some extensions along the glomerular basement membrane. Occasionally, granular (Fig. 2) and linear staining for $\mathrm{IgM}$ and $\lg G$ are reported as seen along the capillary walls ${ }^{41,43}$. On the ultrastructural level, large electrondense deposits are observed in the mesaririum and subendothelial as well as subepithelia!. Moreover, striking changes of the glomerular-endothelial cells are seen $^{38,46}$ (Fig. 3).

Several hypotheses on the pathogenesis of the glomerular lesions exist. Most studies postulate a pathogenetic role for immune complexes as with the other parasite-related glomerulopathies. In some studies, these immune complexes consist of trypanosonal antigen and antibodies 37,42 , but immune complexes comprising autoantibodies and autoantigens have also been implicated 40,47 , especially DNA-antiDNA complexes, as in lupus nephrit:s. together with complement activation $36,38,40$. Others suggested a pathogenetic role for antibodies directed against glomerular autoantigens ${ }^{41,43}$, in the context of polyclonal B-cell activation. Indeed, antibodies against laminin, as well as various glomerular glycoproteins were detected not only in serum, but also in glomerular eluates. Glomerular complement activation was only observed after long-standing infection. Antibodies alone, however, could not account for the development of albuminuria ${ }^{48}$. Host-related factors, determined by nonMHC (major histocompatibility complex) genes were shown to be important for the development of glomerular disease associated with African trypanosomiasis. To delineate which parts of the defence system (in addition to the B-cell response) were involved in the development of glomerular disease, the participation of thymus, spleen and the mononuclear phagocytic system (MPS) were investigated ${ }^{+9}$. It was shown that glomerular disease developed independent of the presence of thymic tissue. Nude mice infected with $T$. brucei parasies developed albuminuria, and some glomerular immunoglobulin deposits. Polyclonal B-cell activation, however, was not observed. Autoantibodies or immune complexes in the context of polyclonal B-cell activation alone, once more, could not explain the rull glomerular disease, ic. histological glomerular changes and albuminuria. In these studies, the spleen was shown to be a crucial organ; albuminuria conild be prevented or significantly lowered, depending on the moment of splenectomy. After macrophage depletion, infected mice developed significantly higher albuminuria for a period up to two weeks after depletion. Therefore, it was concluded that development of glomerulopathy associated with African trypanosomiasis is inciependent of thymusmatured $T$ cells, while mactophages have an inhibitory, rather than inducing, effect. The spleen enlargement during $T$. brici infection is not fully understood. It is, in part, due to an increase in extramedullary hematopoiesis and, in part, due to a proportional increase in $\mathrm{CD}^{+} \mathrm{T}$ cells, $\mathrm{CD} 8^{+} \mathrm{T}$ cells, and $B$ cells. Moreover, a relative increase in null cells (CD4 , CD8 - Ig ) is observed"). In this respect, the massive increase in splenic $\gamma \delta \mathrm{T}$ cells is a particularly interesting finding (B. de Geus it al., Abstracl ${ }^{*}$ ), especially so since these $\gamma \delta \mathrm{T}$ cells seem to be involved in the resistance against $T$. bruco infection ${ }^{51}$. Their role in the pathogenesis of this glomerulopathy should be investigated.

\section{General considerations}

Clinical and epidemiologic studies have established the existence of glomerular disease associated with parasitic infections. Explanation of the pathogenetic mechanism involved, however, remains a difficult challenge. The parasitic infections described above are usually severe chronic infections with fluctuations

\footnotetext{
* Abstract bcok of the Xllth meeting of the International Society of Nephrology, involvement of $\gamma \delta T$ cells in Trypanosomiasis-induced Glomerulopathy: p. 58, 1995
} 
in antigenemia and therefore fluctuations in host immune response. This response consists of several facets that might be nephritogenic.

The antibody response is the most obvious part of the immune system that might act as a 'pathogen', because in most instances glomerular immunoglobulins are found. Theoretically these immunoglobulins might accumulate in glomeruli due to passive trapping or specific binding. The variations in antigenemia during these parasitic infections are encountered by a fluctuating host antibody response, resulting in circulating immune complexes, which might be trapped in the glomerulus, as the BSA-anti-BSA immune complexes in the experiments of Germuth ${ }^{52}$ and Dixon et al.53 According to the size and charge of these complexes, this might result in the different glomerular immunofluorescence staining patterns seen in infected individuals. In experimental malaria infection, however, parasitic antigens were detected in glomeruli before immunoglobulins. This suggisted in situ immune-complex formation. Indeed, Fleuren et al. ${ }^{54}$ showed that alternate perfusion of isolated kidneys with antigen (BSA) and antibody (anti-BSA) can result in in situ formation of glomerular immune complexes, by binding of antibody to the antigen that was 'planted' before. Both theories might explain the presence of immunoglobulins and parasitic antigens in glomer.li as cescribed in several parasitic infections. In many instances, however, parasitic antigen cannot be detected. This might be due to masking of these antigens by antibodies, or clue to the lack of adequate detection methods. Another explanation would be that the glomerular immune complexes contain very little or no parasitic antigen, and thus antihodies with different specificitie: are involved. In this respect, it is of interest that during most of these infections, polyclonal B-cell activation is observed ${ }^{14,55,70}$. The autoantibodies ensuing from this polyclonal activation might form complexes with their antigen and bind in the glomerulus according to the pathways described above. This mechanism was proposed in several papers mentioned ${ }^{15.28}$. Another mechanism involving polyclonal B-cell activation might be direct binding of autoantibodies to glomerular autoantigens, as demonstrated in experimental trypanosomiasis ${ }^{11,43}$. In these studies, antibodies against parasitic antigens as well as antibodies against known nephritogenic autoantigens, such as laminin and dipeptidylpeptidase IV, were eluted from the glomerular immune deposits, and were therefore thought to be pathogenetic. Later studies, however, demonstrated that these antibodies were neither sufficient nor necessary ${ }^{48,44}$ for the development of proteinuria, one of the parameters of glomerular dysfunction. Similar observations were made in other experimental models for glomerulonephritis ${ }^{57,5 x}$. These observations affirmed the importance of other parts of the defence system in the pathogenosis of glomerular disease.

Although most studies on the pathogenesis of glomerulonephritis have, until recently, focused on the characterization of the glomerular imritune deposits, there is now evidence that $T$ cells play a major role in glomerular injury. The potential effect of $T$ cells in glomerular injury was shown to be twofold ${ }^{50}$. While $\mathrm{CD} 4{ }^{+} \mathrm{T}$ cells were shown to be crucial in the induction of the autoimmune syndrome accompanied by 
polyclonal B-cell activation, CD8 ${ }^{+} \mathrm{T}$ cells infiltrating the glomerulus were shown to be directly involved in the onset of proteinuria. Therefore, it is of interest that part of the glomerular hypercellularity in murine malaria is due to influx of $\mathrm{CD}^{+}$cells $^{14}$. Future studies should elucidate the role of $\mathrm{T}$ cells in glomerular injury associated with parasitic infections.

With the exception of B cells and T cells, monocytes and polymorphonuclear leukocytes have been implicated in the pathogenesis of glomerular disease ${ }^{60,61}$, especially in acute glomerulonephritis. The increased celitularity of glomeruli in rabbits infected with African trypanosomiasis was shown to be largely due to infiltration with monocytes and macrophages ${ }^{38}$. As T cells, these components of the primary defence system act through an intricate network of cytokines and other inflammatory mediators such as oxygen radicals ${ }^{62,63.6}$. These mediators might be implicated in cases of glomerular disease without cellular influx as well, especially in parasitic infections where levels of these factors can be presumed to be high due to severe chronic infection and hepatic failure. Natural killer cells or $\gamma \delta \mathrm{T}$ cells might prove to be components of the primary defence system ${ }^{n+, 65}$ and a source of nephrotoxic serum products.

Although the association of parasitic infections with glomerular lesions is clear, several theories on the pathogenesis of these lesions exist. Knowledge on the pathogenetic mechanisms involved has evolved with the knowledge on the pathogenesis of glomerular disease in general. Especially experimental African trypanosominsis has shown that immune complexes are involved but can not explain full-blown glomerular disense. Future studies should elucidate the role of other parts of the defence system in the pathogenesis of glomerular disease associated with parasitic infections.

\section{Acknowledgements}

Thanks we due to Sindine floriun and Phitp / focdemacke for

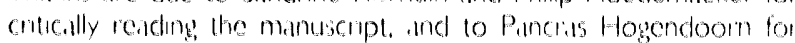
support. The studies described in this wotk were cirriced oul at the Depatment of Pithology, at the Unversty of leder, The Nethetlinds.

\section{Referonces}

I Chugh, K.S. and Sakluuja, V. (199()) Glomerular disease in the tropics. Am. I. Nipurul. 10, 4.37-45(1)

2 Shaper, A.C. (1968) Immunological studies in a tropical environment. Eist Afr. Mill. $1.45,21^{\circ}-228$

3 Fanning, M.M. and Kazura, I.W. ( 9444$)$ Genetic linked variation in susceptibility of mice to schistosomiasis mansoni. Parisiti lmmminol. 6, 95-10)3

4 Cighlioli, C. (I)(2) Malaria and renal disease with special reference to British Guiana. Amm. Trop. Mat. Parnsitul. 56, 225-241

5 Kibukimusoke, J.W., Hutt, M.S.R. and Wilks, N.E. (1967) The nephrotic syndrome in Uganda and is association with quartan malaria. (.) 1. Mit. 36, 39)3-408

6 Boonpucknavig, V. and Sitprija, V. (1979) Renal disease in acute Plasimodium falciparmu infection in man. Kithn'y lut. 16, 44-52

7 Houba, V. (!979) Immunologic aspects of renal lesions associated with maliria. Kituc'l Int. 16, 3-8

\& Sitprija, V. and Boompuiknavig, V. (1994) Renal Pathologery arith Clinical and Functional Sorrelations (Tisher, C.C. and Brenner B.M., edis), pp 626-657, I.P. Lippincott

9 Ward, P.A. and Kibukan'isoke, J.W. (1969) Evidence for soluble immune complexes in the pathogenesis of the glomerulonephritis of quartan malaria. Lancet i. 283-285

10 MzGregor, I.A. ct al. (1968) Soluble antigens in the blood of African patients with severe Plasmodium falciparmm infection in man. Lanct' $i, 881-884$

11 Boonpıcknavig, S., Boonpucknavig, V. and Bhamarapravati, N. (1972) Immunopathological studies of Plasmodium bergheiinfected mice. Arch. Pathol. 94, 322-330
12 Parbtani, A. and Cameron, J.S. (1979) Experimental nephritis associated with Plasmodium infection in mice. Kidn'y lnt. 16, $53-63$

13 Ehrich, J.H. et al. (1981) Rat malarial glomerulonephritis. An experimental model of post-infectious glomerular injury. Virchous Arch. IBI 37, 343-356

14 Lloyd, C.M., Wozencraft, A.O. and Williams, D.G. (1993) Cellmediated pathology during murine malaria-associated nephritis. Clin. Exp. Immmunol. 94, 398-402

15 Wozencraft, A.O. ' $t$ ( 1 l. (1990) Role of DNA-binding antibodies in kidney pathology associated with murine malaria infections. Infect. Inmmint. 58, 2156-2164

16 Andrade, Z.A., Andrade, S.G. and Sadigurskv, M. (1971) Rena? changes in patients with hepatosplenic schistosomiasis. $A m$. J. Trop. Med. Hyg. 20, 77-83

17 Rocha, $H$. it al. (1976) Renal involvement in patients with hepatosplenic schistosomiasis mansoni. Am. I. Trop. Mct. Hyg. 25, 108-115

18 Barsoum, R.S. 't al. (1977) Renal disease in hepatosplenic schistosomiasis: a clinicopathological study. Trtuts. $R$. Soc. Trop. Med. Hy. $71,387-391$

19 Barsoum, R.S. (1993) Schistosomal glomerulopathies. Kithey lit. 44, 1-12

20) Andrade, Z.A. and Rocha, H. (1979) Schistosomal glomerulopathy. Kidu'y Int. 16, 23-29

21 Sobh, M.A. it al. (1987) Schistosomal specific nephropathy leading to end-stage renal failure. Kithey lnt. 31, 1006-1011

22 Hoshino-Shimizu, S. if al. (1976) Human schistosomiasis: Schistosoma mansoni antigen detection in renal glomeruli. Trums. R. Suc. Trop. Ned. Hys. 70, 492-496

23 van Marck, E.A.E., Deelder, A.M. and Gigase, P.L.J. (1977) Effect of portal vein ligation on immune glomerular deposits in Sclistosoma mansoni infected mice. Br. J. F.y. Pathol. 58, 412-417

24 Houba, V. (1979) Experimental renal disease due to schistosomiasis. Kittu'y!nt. 16, 30-43

25 Martinelli, R. it al. (1989) Schistosoma mansoni-induced mesangiocapillary glomerulonephritis: influence of therapy. Kitili'ly lint. 35, 1227-1233

26 Sobh, M.A. 't al. (1988) Effect of anti-schistosomal treatment on schistosomal-specific nephropathy. Niphlowl. Diol. Trmmspliml. 3, $7+4.4-751$

27 Civallo, T. it $111 .(1974)$ The nephropathy of experimental hepatosplenic schistosomiasis. Am. I. Pothol. 76, 433-450

28 Hillyer, C.V. and Lewert, R.M. (|47,t) Studies on renal pathology on hamsters infected with Schisfosomn monsomi and

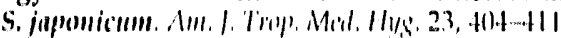

2) Fujiwata, M., Makinu, M. and Walanube, H. (1488) Sihistosanna mansoni: induction of severe glomerulomephritis in female BXSB mice following chronic infection. Exy. I'musiful. 65, $2|+-22|$

30) Barsum, R.S. it al. (1988) Hepatic macrophage function in schistosomal glomerulopathy. Nephnol. Diul. Trmisplant. 3, 612-6)16

31 de Brito, T. ('t al. (1975) Glomerular involvement in human kalaazar: $A$ light, immunofluorescent and electron microscopic study based on kidney biopsies. Am. I. Trop. Me't. Hys. 24, 9-18

32 Sarturi, A. c't al. (1991) Immune complex glomerulonephritis in experimental kala-azar II: Detection and characterization of parasite antigens and antibodies eluted from kidneys of Leis/mmania donovani-infected hamsters. Clin. Exp. Immmuml. 87, $38(1-392$

33 Dutra, M. ct al. (1985) Renal involvement in visceral leishmaniasis. Am. I. Kitlucy Dis, 6, 22-27

34 Oliveira, A.V. it al. (1985) Mesangial proliferative glomerulonephritis associated with progressive amyloid deposition in hamsters experimentally infected with Leishmmuia donovmin. Am. I. Puthol. 120), 250-262

35 Sartori, A. 't al. (1987) Immune complex glomerulonephritis in experimental kala-azar. Pinnsite Immunol. 9, 93-103

36 Nagle, R.B. ct atl. (1974) Experimental infections with African trypanosomes. VI Glomerulonephritis involving the altentive pathway of complement activation. Am. J. Trop. Mod. Hyg. 23, 15-26

37 Facer, C.A. c' al. (1973) Trypanosoma brucei: renal pathology in rabbits. Exp. Parasitol. 44, 249-261

38 Nagle, R.B. ct al. (1982) Glomerular accumulation of monocytes and macrophages in experimental glomerulonephritis associated with Trypanosoma rhodesiense infection. Lab. Ini'st. 46, 356-376

39 Lindsley, H.B. it al. (1980) Variable severity of glomerulonephritis in inbred rats infected with Trypanosoma rhodesiense. 
Correlation with immunoglobulin class-specific antibody responses to trypanosomal antigens and total IgM levels. Am. I. Trop. Med. Hyg. 29, 348-357

40 Rickman, W.J and Cox, H.W. (1979) Association of autoantibodies with anemia, splenomegaly, and glomerulonephritis in experimental African trupanosomiasis. J. Parasilol. 65, 65-73

41 Bruijn, J.A. ct al. (1987) Anti-basenient membrane glomerulopathy in experimental trypanosomiasis. I. Immunnol. 139, 2482--2488

42 van Marck, E.A.E. ct al. (1981) Renal disease in chronic experimental Trypanosoma ganbiense infections. Am. J. Trop. Mied. Hyg. 30, 780-789

43 van Velthuysen, M-L.F. et al. (1992) Pathogenesis of trypanosomiasis-induced glomerulonephritis in mice. Nephlrol. Dial. Transplant. 7, 507-515

44 Barrett-Connor, E., Ugoretz, R.G. and Braude, A.L. (1973) Disseminated intravascular coagulation in trypanosomiasis. Arch. Intern. Med. 131, 574-577

45 Costa, R.S. it al. (1991) Immune complex-mediated glomerulopathy in experimental Chagas' disease. Clin. Immtunol. Immintopathol. 58, 102-114

46 van Velthuysen, M-L.F. ef al. (1994) Phagecytosis by glomerular endothelial cells in infection-related glomerulopathy. Nephrol. Dial. Transplant. 9, 1077-1083

47 Lindsley, H.B., Nagle, R.B. and Stechschulte, D.J. (19,8) Proliferative glomerulonephritis, hypocomplementemia, and nucleic acid antibodies in rats infected with Trypanosoma rhodesiense. Ail. I. Trop. Med. Hyg. 27, 865-872

48 van Velthuysen, M-L.F. c't al. (1993) Susceptibility for infectionrelated glomerulopathy depends on non-MHC genes. Kidul'y lnt. 43, 623-629

49 van Velthuysen, M-L.F. et a! (1994) T cells and macrophages in Trypanosoma brucei-related glomerulopathy. Infecl. Immmin. 62, 3230-3235

50 Makumyaviri, A.M. it al. (1988) Sj'steme lymphocytaire et resistance relative de la souris consanguine a Trypanosomo brucei brucei. Ann. Inst. Pastent lmmunol. 139, 545-556

51 Flynn, J.N. and Sileghem, M.R. (1994) Involvement of gammadelta $\mathbf{T}$ cells in immunity to trypanosomiasis. Immmunology 83 , $86-92$

52 Germ: in, F.G. (1953) A comparative histologic and immuno- logic study in rabbits of induced hypersensitivity of the serum sickness type. J. Exp. Med. 97, 257-282

53 Dixon, F.J., Feldman, I.D. and Vazquez, J.J. (1961) Experimental glomerulonephritis. The pathogenesis of a labuadtory model resembling the spectrum of human glomerulonephritis. I. Exp. Met. 113, 899-920

54 Fleuren, G.J., Grond, J. and Hoedemaeker, P.J. (1980) In situ formation of subepithelial glomerular immune complexes in passive serum sickness. Kidn'y Int. 17, 613-637

55 Kobayakawa, T. et al. (1979) Autoimmune responses to DNA red blood cells, and thymocyte antigens in association with polyclonal antibody sythesis during experimental African trypanosomiasis. I. Immuntol. 122, 296-301

56 Hentati, B. et al. (1994) Beneficial effect of polyclonal immunoglobulins from malarie-infected BALB/c mice on the lupuslike syndrome of (NZB X NZW)F1 mice. Eur. J. Immuntol. 24, 8-15

57 Aten, J. et al. (1988) Cyclosporin A induces long-term unresponsiveness in mercuric chloride-induced autoimmune glomerulonephritis. Clin. Exp. Immunol. 73, 307-311

58 Kawasaki, K. et al. (1992) Depletion of CD8 positive cells in nephrotoxic serum nephritis of WKY rats. Kidn'y lnt. 41 1517-1526

59 Florquin, S. and Goldman, M. (1994) T cell subsets in glomerular diseases. Springer Semin. Immmunopathol. 16, 71-80

60 Cattell, V. (1994) Macrophages in acute glomerular inflammation. Kidn'y Int. 45, 945-952

61 Schrijver, G. it al. (1990) Anti-GBM nephritis in the mouse: role of granulocytes in the heterologous phase. Kidney $\mathrm{ht}$. 38, 86-95

62 Benigni, A. and Remuzzi, G. (1994) Inflammation and glomerular injury. Springer Semin. Immmmonthol. 16, 39-51

63 Diamond, J.R. (1992) The role of reactive oxygen species in animal models of glomerular disease. Am. I. Kitu'y Dis. 19, 292-300

64 Scharton, T.M. and Scott, P. (1993) Naturai killer cells are a source of interferon gamma that drives differentiation of $\mathrm{CD}_{4}$ $T$ cell subsets and induces early resistance to Leishmania major in mice. I. Exp. Met. 178, 567-577

65 Hiromatsu, K. ct al. (1992) A protective role of gamma-delta T cells in primary infection with Listeria monocytogenes in mice. J. E.xp. Mcit. 175, 49-56

\section{Recent articles in other Trends journals}

The role of nuclear import and export in influenza virus infection, by G. Whittaker; $M$. Bui and A. Helenius (1996) Trends in Cell Biology 6, 67-71

The contrasting roles of $\mathrm{CD4}^{+} \mathrm{T}$ cells in intracellular infections in humans: leishmaniasis as an example, by M. Kemp, T.G. Theander and A. Kharazmi (1996) Immunology Today 17, 13-16

Rhodococcus equi: an emerging opportunistic pathogen, by D.M. Mosser and M.K. Hondalus (1996) Trends in Microbiology 4, 29-33

Programmed DNA deletions in Tetrahymena: mechanisms and implications, by M-C. Yao (1996) Trends in Genetic: 12, 26-30

Sacrifice in the face of foes: pathogen-induced programmed cell death in plants, by $R$. Mittler and E. Lam (1996) Trends in Microbiology 4, 10-15

Communicate your science! Super seminar slides, by B.S. Brown (1996) Trends in Cell Biology 6, 74-76

\section{Coming soon in Parasitology Today}

DNA replication in the malaria parasite, by J.H. White and B.J. Kilbey

Genomic repetitive elements of Trypanosoma cruzi, by J.M. Requena, L.C. Lopez and C. Alonso

Modulation of host cell intracellular $\mathrm{Ca}^{2+}$, by $\mathrm{M}$. Olivier

Lymphatic filariasis: what mice can tell us, by R.A. Lawrence

Currently available molluscicides, by S. Perrett and P.J. Whitfield

$\gamma / \delta$ T cells in malaria infections, by J. Langhorn

Cryptosporidium parvum and our water supply, by G. Widmer, M. Carraway and S. Tzipori

Mosquitocidal toxins, genes and bacteria: the hit squad, by A.G. Porter

Don't miss these, and many other articles of interest: subscribe to Parasitology Today using the form bound in this issue 